\title{
Clinical, radiologic and serologic profile of patients with interstitial pneumonia with autoimmune features: a cross-sectional study
}

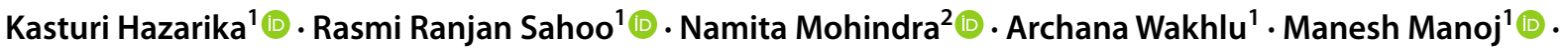

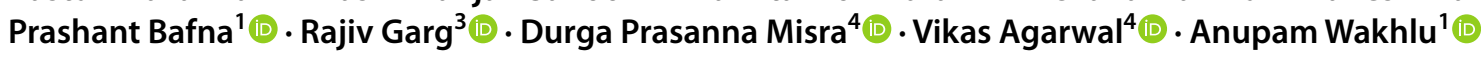

Received: 11 March 2021 / Accepted: 30 April 2021 / Published online: 27 May 2021

(c) The Author(s), under exclusive licence to Springer-Verlag GmbH Germany, part of Springer Nature 2021

\begin{abstract}
The current study aimed to characterize patients from a rheumatology referral center in north India, who satisfied the definition of interstitial pneumonia with autoimmune features (IPAF) as given by the American Thoracic Society and European Respiratory Society (ATS/ERS) consensus committee in 2015. Thirty-five adult patients aged 18 years and above, fulfilling the 2015 ATS/ERS criteria for IPAF were included in the study. The clinical and immunological profile, and radiologic findings on high-resolution computerized tomography thorax were noted. Antinuclear antibody (ANA) by indirect immunofluorescence at 1:320 titer and myositis-specific antibody (MSA) assays were performed. Non-parametric tests were used to compare variables between groups. The study cohort included predominantly female patients with a mean age of $50.6 \pm 13$ years and mean duration of disease of $38.8 \pm 28.4$ months. Majority of patients (49\%) fulfilled the morphologic and serologic domains as per the IPAF consensus criteria and $31 \%$ patients had features in all three domains. Non-specific interstitial pneumonia was the most common pattern observed in $77 \%$ patients. Raynaud's phenomenon and inflammatory arthritis were the predominant autoimmune features. Pulmonary arterial hypertension was documented in $60 \%$ of patients on echocardiography. Positive ANA at 1:320 dilution was present in all 26 patients tested, whereas extractable nuclear antigen and MSA assays detected autoantibodies in $49 \%$ and $51 \%$ of patients respectively. IPAF predominantly affected females in the age group of 50 years and above, with varied autoimmune manifestations and autoantibody profile.
\end{abstract}

Keywords Idiopathic interstitial pneumonias · Interstitial lung disease $\cdot$ Autoantibodies · Connective tissue diseases

\section{Introduction}

Interstitial lung disease (ILD) or diffuse parenchymal lung disease (DPLD) encompasses a group of disorders involving the lung interstitium occurring due to various causes. The known causes include a variety of connective tissue diseases (CTD), drugs and environment-related factors. Those

Anupam Wakhlu

anupamwakhlu@gmail.com

1 Department of Clinical Immunology and Rheumatology, King George's Medical University, Lucknow 226003, India

2 Department of Radiodiagnosis, Sanjay Gandhi Post Graduate Institute of Medical Sciences, Lucknow 226014, India

3 Department of Respiratory Medicine, King George's Medical University, Lucknow 226003, India

4 Department of Clinical Immunology and Rheumatology, Sanjay Gandhi Post Graduate Institute of Medical Sciences, Lucknow 226014, India without a definite cause are termed idiopathic interstitial pneumonias (IIP), which constitutes a distinct entity, causing damage to the lung parenchyma with varying patterns of inflammation and fibrosis. IIP is classified into seven distinct patterns, based on histologic and radiologic features [1]. Except for usual interstitial pneumonia (UIP), the other patterns of lung involvement, especially non-specific interstitial pneumonia (NSIP), lymphocytic interstitial pneumonia (LIP), cryptogenic organizing pneumonia (COP), and acute interstitial pneumonia (AIP), demand an extensive search into their possible etiologies, requiring multidisciplinary attention from radiology, pathology, rheumatology, and pulmonology specialties, with important therapeutic implications.

The connective tissue diseases-rheumatoid arthritis (RA), systemic sclerosis ( $\mathrm{SSc}$ ), idiopathic inflammatory myositis (IIM), Sjogren's syndrome (SS), systemic lupus erythematosus (SLE) and mixed connective tissue disease (MCTD) have long been known to be associated with ILD 
that responds favourably to immunosuppression. However, it is not uncommon for patients with IIP to present with certain autoimmune features, either in the form of autoantibodies or with some clinical features, that do not fulfill the criteria for a definite CTD [2,3]. These group of disorders have been variably called lung-dominant connective tissue disease and autoimmune ILD in the past [4-6].

The joint committee by American Thoracic Society and European Respiratory Society (ATS/ERS) in 2015 released an official statement designating the term interstitial pneumonia with autoimmune features (IPAF) to describe patients with ILD, who do not meet classification criteria for a defined CTD but have some possible clinical features of an autoimmune disease [7]. This has brought in a homogeneity to this group of patients with ILD and autoimmune features, not fitting into a definite CTD, encouraging further studies to look into its pathophysiologic aspects, treatment options, and outcomes.

The current study aimed to determine the clinical, radiologic, and serologic features of IPAF patients from a rheumatology referral centre in north India. It also compares the findings with other similar studies and adds a rheumatologist's perspective to the same.

\section{Methods}

This cross-sectional study included adult patients of IIP of age 18 years or above, fulfilling the 2015 ATS/ERS classification criteria for IPAF, and attending the departments of Respiratory medicine and Rheumatology of a tertiary care center in north India from August 2019 to September 2020 over a period of 1 year. Patients qualifying as definite CTD as per the respective classification criteria or as sarcoidosis, based on presenting features, were excluded. Those with lung involvement attributable to a history of drug or environmental exposure were also excluded [8-12]. Ethical clearance was obtained from the institutional ethics committee and the study was conducted as per the Helsinki Declaration and good clinical practice guidelines. A written informed consent was taken from all participants.

All patients were subjected to detailed history, thorough physical examination, and underwent routine and special immunological tests. Rheumatoid factor (RF) was done by turbidimetry and value more than two times upper limit of normal (ULN) was considered abnormal, as per the ATS/ ERS consensus criteria for IPAF. Anti-cyclic citrullinated peptide (CCP) was measured by enzyme-linked immunosorbent assay (ELISA) and values more than the ULN were considered positive. Antinuclear antibody (ANA) was performed by indirect immunofluorescence (IIF) technique using HEp- 2 cells at both 1:100 and 1:320 dilutions, and patterns and intensity of fluorescence were noted.
Extractable nuclear antigen (ENA) and myositis-specific antibody (MSA) assays were done by immunoblot technique according to manufacturer's instructions (Euroimmun, Germany).

The diagnosis of interstitial pneumonia and the pattern of involvement was assessed by high-resolution computerized tomography (HRCT) of thorax, which was preferably obtained at $1-\mathrm{mm}$ thin slices by volumetric acquisition. The films were independently read by two radiologists and consensus opinion was taken whenever deemed appropriate. Severity of lung involvement was based on visual estimation as decided by the radiologists and categorized as mild, moderate and severe.

Pulmonary function test (PFT) was done to assess the forced expiratory volume (FEV1), forced vital capacity (FVC) and diffusion lung capacity for carbon monoxide (DLCO). Pulmonary artery hypertension (PAH) was defined as a mean pulmonary artery pressure (mPAP) of $20-\mathrm{mm} \mathrm{Hg}$ or more at rest [13]. The mPAP was derived from the pulmonary acceleration time (PAT) measured on two-dimensional echocardiogram (Echo) [14].

\section{Statistical analysis}

Statistical analysis was done using Graph pad Prism 5.01 (GraphPad Software, San Diego, CA, USA). Quantitative data were expressed as mean and standard deviation, whereas qualitative data as frequency and percentage. Mann-Whitney and Fisher's exact tests were used to compare numerical and categorical variables between groups respectively. $P$ value of $<0.05$ was considered statistically significant.

\section{Results}

\section{Demographic characteristics}

There were 35 adult patients ( 29 females) with a mean age of 50.6 \pm 13.1 years (range 25-75 years). Majority (54\%) of the patients were older than 50 years. The mean BMI of patients was $23.4 \pm 4.3 \mathrm{~kg} / \mathrm{m}^{2} ; 51 \%$ were overweight or obese (BMI $\geq 23 \mathrm{~kg} / \mathrm{m}^{2}$ ).

\section{Clinical profile of patients}

The mean duration of disease was $38.8 \pm 28.4$ months, with $63 \%$ of patients presenting between 1 and 5 years of disease onset. Among the autoimmune features, inflammatory arthritis and Raynaud's phenomenon (RP) were common (31\% each). One patient had mechanic's hand. Autoimmune features, which are not included in the ATS/ERS 2015 IPAF guidelines, were also recorded. Subclinical myositis was present in two patients, photosensitive skin rash in one patient 
and two patients had a non-specific papular skin rash. Periarticular calcinosis around proximal and distal interphalangeal joints was seen in one patient. Recurrent oral ulcers and periungual cuticular changes were present in one patient each. Two patients had immune thrombocytopenia and one patient was receiving treatment for autoimmune hemolytic anemia (AIHA). The frequency of various IPAF features in the study cohort are shown in Table 1.

\section{Radiologic characteristics of patients}

The various radiologic findings on HRCT thorax were ground glass opacities (GGO), reticulation and fibrosis, traction bronchiectasis (TB), honey combing (HC), and cysts. GGO was the most common abnormality (89\%), followed by reticulation and fibrosis ( $83 \%)$. The combination of GGO and fibrosis was present in 7 out of 35 patients (20\%), whereas 17 patients (49\%) had GGO, fibrosis and TB. NSIP pattern on HRCT was diagnosed in $27(77 \%)$ patients, of which 19 patients (54\%) had fibrotic NSIP. Seven out of 35 patients (20\%) had UIP pattern and satisfied both the clinical and serologic domains as per the IPAF definition. LIP was recorded in only one patient. The clinical, radiologic, and serologic features of different groups according to severity of lung involvement on HRCT are summarized in Table 2.

Figure 1 depicts the clinical and radiologic features of patients observed in the study cohort.

\section{Prevalence of PAH}

Twenty-one out of 35 patients (60\%) had PAH as measured on echocardiography. Majority (43\%) had a mild PAH with mPAP $\leq 40-\mathrm{mm}$ Hg. Pulmonary artery dilatation, defined by pulmonary artery: aorta ratio of more than one on HRCT, was noted in seven patients (20\%). Of the 28 patients whose pulmonary artery size appeared normal on HRCT thorax, $14(50 \%)$ were diagnosed with PAH on echocardiography.

\section{Immunological profile of patients}

$\mathrm{RF}$, more than two times ULN, was present in one patient (3\%), whereas ACPA was positive in five patients $(14 \%)$. Thirty-four out of 35 patients (97\%) were positive for ANA by immunofluorescence at 1:100 dilution, whereas all 26 patients for whom ANA could be done at 1:320 dilution, showed a positive pattern. ENA and MSA assays detected autoantibodies in $49 \%$ and $51 \%$ of patients, respectively, with anti-Ro52 and anti-tRNA synthetase being the most common. The clinical and radiological characteristics of patients with anti-Ro52 and anti-tRNA synthetase antibodies are compared in Table 3. Other laboratory details of patients are provided in Supplementary Table 1.

Table 1 Different IPAF features in the study cohort $(N=35)$

\begin{tabular}{llll}
\hline Clinical domain & $N(\%)$ & Serologic domain & $N(\%)$ \\
\hline Distal digital fissuring & $1(3)$ & ANA* & 26(100) \\
Distal digital tip ulceration & 0 & RF $\geq 2$ ULN & $1(3)$ \\
Inflammatory arthritis or polyarticular morning joint & $11(31)$ & Anti-CCP & $5(14)$ \\
$\quad$ stiffness & & Anti-dsDNA & $1(3)$ \\
Palmar telangiectasia & 0 & Anti-Ro (SS-A) & $11(31)$ \\
Raynaud's phenomenon & $11(31)$ & Anti-La (SS-B) & $1(3)$ \\
Unexplained digital edema & 0 & Anti-ribonucleoprotein & $1(3)$ \\
Gottron sign & 0 & Anti-Smith & $1(3)$ \\
& & Anti-topoisomerase (Scl-70) & $4(11)$ \\
& & Anti-tRNA synthetase & $7(20)$ \\
\hline Morphologic domain & & & \\
\hline Suggestive radiology patterns by HRCT & & & \\
NSIP & & & \\
OP & & & \\
NSIP with OP overlap & & & \\
LIP & & & \\
UIP & & & \\
Multi-compartment involvement in addition to interstitial pneumonia & & \\
\hline
\end{tabular}

$I P A F$ Interstitial pneumonia with autoimmune features, $A N A$ Antinuclear Antibody, $R F$ Rheumatoid factor, $C C P$ cyclic citrullinated peptide, $D N A$ deoxyribonucleic acid, SSA/B Sjogren's syndrome antigen A/B, HRCT high resolution computerized tomography, NSIP Non-specific interstitial pneumonia, OP Organizing pneumonia, LIP Lymphoid interstitial pneumonia; *ANA by immunofluorescence at 1:320 dilution was done in 26 patients 
Table 2 Comparison of clinical and radiographic characteristics among patients with mild, moderate, and severe disease-based extent of lung involvement on HRCT $(N=35)$

\begin{tabular}{|c|c|c|c|}
\hline & Mild $(n=9)$ & $\begin{array}{l}\text { Moderate } \\
(n=17)\end{array}$ & Severe $(n=9)$ \\
\hline Age in years & $45(34-53.5)$ & $55(48.5-65)^{*}$ & $45(39-61.5)$ \\
\hline Male/female, $(n)$ & $1 / 8$ & $4 / 13$ & $1 / 8$ \\
\hline DOI, months & $24(15-48)$ & $26(15-60)$ & $48(24-75)$ \\
\hline \multicolumn{4}{|l|}{ HRCT pattern, $(n)$} \\
\hline NSIP & 8 & 13 & 6 \\
\hline UIP & 1 & 3 & 3 \\
\hline LIP & 0 & 1 & 0 \\
\hline $\mathrm{PAH},(n)$ & $4 / 9$ & $9 / 17$ & $8 / 9^{\#}$ \\
\hline FVC & $67.5(45-86.5)$ & $64(53-68.5)$ & $-\#$ \\
\hline FEV1/FVC & $\begin{array}{l}106(101.3- \\
108.8)\end{array}$ & $112(97-118.5)$ & $-\#$ \\
\hline DLCO & $38.5(27.5-63)$ & $40(36-49)$ & $-\#$ \\
\hline \multicolumn{4}{|c|}{ Autoimmune features, $(n)$} \\
\hline Arthritis & 5 & 7 & 0 \\
\hline Raynaud's & 3 & 3 & 4 \\
\hline Skin rash & 2 & & 0 \\
\hline Mechanic hands & 0 & 1 & 0 \\
\hline $\begin{array}{l}\text { Subclini- } \\
\text { cal myositis }\end{array}$ & 1 & 1 & 0 \\
\hline \multicolumn{4}{|c|}{ Serologic features, $(n)$} \\
\hline ANA positive & 9 & 16 & 9 \\
\hline $\begin{array}{l}\text { Anti-CCP posi- } \\
\text { tive }\end{array}$ & 1 & 1 & 3 \\
\hline Anti-Ro52 & 4 & 2 & 4 \\
\hline Anti-Scl70 & 0 & 4 & 0 \\
\hline Anti-tRNA & 2 & 3 & 2 \\
\hline Anti-Mi2B & 1 & 2 & 1 \\
\hline Anti-SRP & 1 & 1 & 1 \\
\hline
\end{tabular}

Data are shown in median and interquartile range when represented

DOI duration of illness, HRCT high-resolution computerized tomography, NSIP non-specific interstitial pneumonia, UIP usual interstitial pneumonia, LIP lymphoid interstitial pneumonia, $P A H$ pulmonary hypertension, $F E V 1$ forced expiratory volume in $1 \mathrm{~s}$ (percentage of predicted value), $F V C$ forced vital capacity (percentage of predicted value), $D L C O$ diffusing capacity of lungs for carbon monoxide (percentage of predicted value), ANA antinuclear antibody, $C C P$ cyclic citrullinated peptide, $S R P$ signal recognition particle

$* p=0.049$ for mild vs moderate disease

\#Only three patients with severe lung disease could perform the pulmonary function tests; hence median values were not calculated in the severe disease

\section{Clinical characteristics of patients with arthritis and Raynaud's phenomenon}

Inflammatory arthritis and RP were the two dominant autoimmune features present in our cohort. They were present concomitantly in four patients. Seven patients, who had only inflammatory arthritis, had mild-to-moderate degree of lung involvement on HRCT and majority (six out of seven patients) had NSIP pattern. None of them were positive for $\mathrm{RF}$ or anti-CCP, but all of them had a positive ANA. Isolated RP was present in seven patients and were found to have moderate-to-severe degree of lung involvement, with both NSIP and UIP patterns. All of them were positive for ANA, and anti-Ro52 was present in three of them. These patients had more severe disease with progression of symptoms despite treatment, with eventual demise of two patients.

The clinical characteristics of patients with and without $\mathrm{RP}$ are compared in Table 4.

\section{Treatment details}

All patients were prescribed steroids. Cyclophosphamide and mycophenolate mofetil were the most common accompanying immunosuppressants used (12 out of 35 each, 34\%). Cyclophosphamide induction was followed by azathioprine for maintenance in three patients, mycophenolate mofetil in two patients and tacrolimus in two patients.

\section{Follow-up}

Four patients expired during the study - one due to Corona virus disease 2019 (COVID-19) pneumonia and the other three due to progression of disease and superadded infective episodes. Six patients were lost to follow-up amidst the pandemic. Thirteen patients were stable during the course of the study. Three patients had progression of symptoms despite treatment, three patients required hospitalizations for pulmonary infections (other than COVID-19) and three patients were on domiciliary oxygen therapy.

\section{Discussion}

The patients who fulfilled the classification of IPAF in the present study were predominantly females and aged 50 years and above, which correlates with similar studies [15-20]. Most patients presenting to our tertiary center had a duration of illness between 1 and 5 years. Avala et al. have reported that IPAF patients were erroneously treated as pulmonary tuberculosis, hypersensitivity pneumonitis or obstructive airway disease [19]. This could be an important reason for late presentation and referral, leading to a delayed diagnosis. Majority of patients in the study cohort fulfilled criteria in the morphologic and serologic domains, whereas serological criteria were fulfilled by all patients.

The prevalence of breathlessness, cough and non-anginal chest pain in the study group was similar to that reported in ILD in general and also in other IPAF cohorts [19, 21]. Inflammatory arthritis and RP were the two most common autoimmune features and have been similarly observed in 

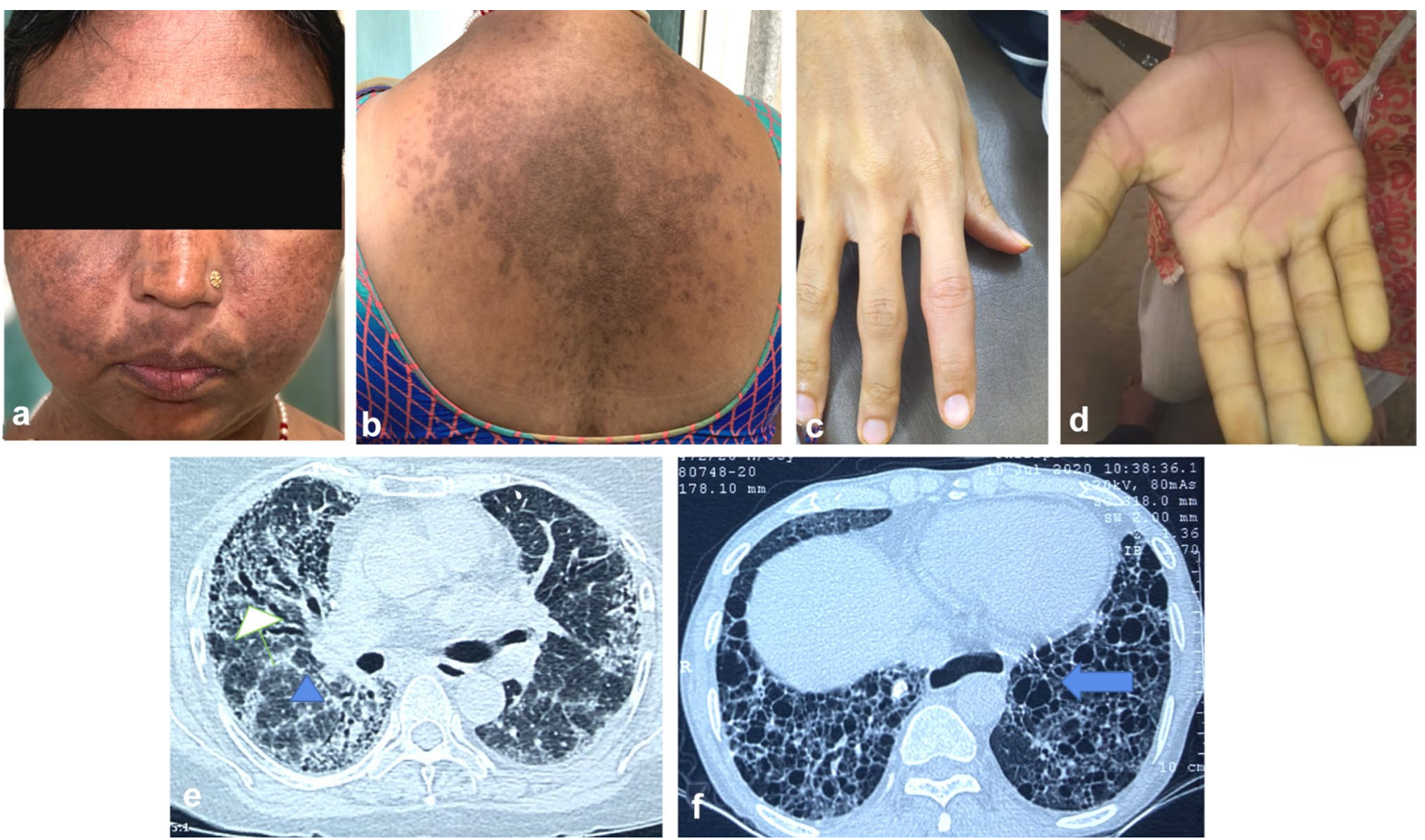

Fig. 1 Clinical and radiologic characteristics of IPAF patients: photosensitive skin rash involving the face sparing the nasolabial fold (a), and over back (b); inflammatory arthritis involving the proximal interphalangeal joints of hand (c); Raynaud's phenomenon (d); ground glass opacities (blue arrowhead) and fibrotic bands with trac-

other studies [15, 19, 22, 23]. Majority of patients with inflammatory arthritis and ILD in the study had a NSIP pattern on HRCT, in contrast to the UIP pattern usually seen in RA [24]. These patients had mild-to-moderate disease and were stable on treatment. Patients with RP had a signification association with the UIP pattern $(p=0.0017)$. Mechanics hand was reported in 28.6\% IPAF patients by Chartrand et al. but was present in only $3 \%$ patients in our cohort [16]. Other IPAF-defining autoimmune clinical features such as digital edema, digital ulcers, Gottron papules and palmar telangiectasia were not seen in our study population. The inclusion of the seven specific autoimmune features in the definition of IPAF, while excluding other clinical features with a possible autoimmune etiology, has not been well rationalized. In an IPAF cohort from Greece, a morbilliform skin rash was reported as a common finding, while another study from Italy reported sicca features as a common association $[23,25]$. Although present in a few cases, other autoimmune features such as photosensitive skin rash, nail cuticular changes, subclinical myositis, history of AIHA and immune thrombocytopenia were encountered in our cohort. The presence of these features, along with positive serology tion bronchiectasis (white arrowhead) as seen on axial section of high-resolution computerized tomography (HRCT) of thorax suggestive of fibrotic non-specific interstitial pneumonia, and (e) and honeycombing and cystic changes (blue arrow) as seen on axial sections of HRCT suggestive of usual interstitial pneumonia (f)

and IIP, could indicate an undifferentiated or evolving connective tissue disorder in these patients.

The presence of NSIP, which was the predominant radiologic pattern in our study, is an important indication to look for an underlying CTD or autoimmune feature, as it predicts alveolar inflammation and possibly warrants immunosuppression. NSIP has also been described as the most prevalent pattern on HRCT and lung biopsy samples in similar cohorts $[16,18,22,26]$.

The presence of UIP pattern alone was not enough to classify patients as IPAF, because of its close resemblance with IPF radiologically. However, it cannot be ignored that UIP is the most common pattern of lung involvement in RA and is also seen, albeit rarely, in patients of scleroderma. In the past, it has been shown that in UIP, bronchoalveolar lavage revealed lymphocytosis and histology revealed cellular inflammation similar to NSIP.

The retrospective studies by Oldham et al. and Sharma et al. reported UIP pattern as the predominant pattern in patients who were subsequently reclassified as IPAF (54.6\% and $35.7 \%$ of patients, respectively) $[15,17]$. In the present study, patients with UIP pattern on HRCT had additional features, both from the serologic and clinical 
Table 3 Comparison of clinical and radiographic characteristics of patients with anti-Ro52 and anti-t-RNA synthetase antibody

\begin{tabular}{lll}
\hline Characteristics & $\begin{array}{l}\text { Anti-Ro52 antibody } \\
(n=10)\end{array}$ & $\begin{array}{l}\text { Anti-tRNA synthetase } \\
\text { antibody }(n=8)\end{array}$ \\
\hline Age in years & $45(35-61)$ & $44(30-51)$ \\
Female/male, $(n)$ & $8: 1$ & $6: 2$ \\
Duration of disease in months & $18(17-24)$ & $48(18-60)$ \\
HRCT pattern, $(n)$ & & \\
NSIP & 7 & 7 \\
UIP & 2 & 0 \\
LIP & 0 & 1 \\
Extent of lung involvement on HRCT, $(n)$ & & 3 \\
Mild & 3 & 4 \\
Moderate & 2 & 1 \\
Severe & 4 & 5 \\
PAH, $(n)$ & 6 & $48(44-79)$ \\
FVC & $70(58.5-90)$ & $103(101-112)$ \\
FEV1/FVC & $109(102-119)$ & $42(28.5-61.5)$ \\
DLCO & $36(32.3-55)$ & Inflammatory arthritis-3 \\
Autoimmune features, $(n)$ & Inflammatory arthritis-2 & Raynaud's-1 \\
& Raynaud's-4 & Subclinical myositis-1 \\
& Recurrent Oral ulcers-1 & Calcinosis-1 \\
Skin rash-1 & \\
Immune thrombocytopenia-1 & & Mi2b-4 \\
& & SRP-1 \\
\hline
\end{tabular}

Data are shown in median and interquartile range when represented

$H R C T$ high-resolution computerized tomography, NSIP non-specific interstitial pneumonia, UIP usual interstitial pneumonia, LIP lymphoid interstitial pneumonia, $P A H$ pulmonary hypertension, $F E V 1$ forced expiratory volume in one second (percentage of predicted value), $F V C$ forced vital capacity (percentage of predicted value), $D L C O$ diffusing capacity of lungs for carbon monoxide (percentage of predicted value), $S R P$ signal recognition particle
Table 4 Comparison of clinical characteristics of patients with and without Raynaud's phenomenon

\begin{tabular}{llll}
\hline & $\begin{array}{l}\text { RP } \\
(n=11)\end{array}$ & $\begin{array}{l}\text { Without RP } \\
(n=24)\end{array}$ & $P$ value \\
\hline PAH & 6 & 15 & 0.72 \\
$\begin{array}{l}\text { Pulmonary artery dilata- } \\
\text { tion on HRCT }\end{array}$ & 2 & 5 & 1 \\
$\begin{array}{l}\text { Anti-Ro52 positive } \\
\text { UIP pattern on HRCT }\end{array}$ & 4 & 6 & 0.68 \\
\hline
\end{tabular}

$R P$ Raynaud's phenomenon, $P A H$ pulmonary artery hypertension, UIP usual interstitial pneumonia

${ }^{*} p<0.05$ considered statistically significant

characteristics, thus fulfilling the criteria for IPAF. LIP, as also reported by Avala et al. was a rare finding. Organizing pneumonia, reported in around $15-30 \%$ by HRCT and surgical lung biopsy in IPAF patients, was not observed in the current study [18, 19, 22].

Extra-thoracic features like unexplained pleural or pericardial thickening, unexplained small airway disease and pulmonary vasculopathy have been included as part of the morphologic domain of the 2015 ATS/ERS definition for IPAF [7]. However, no standard defining features exist for the extra-thoracic morphologic features. In the two cohorts from Japan and Italy, this aspect of multicompartment pathology was overlooked while defining IPAF patients $[23,27]$. Also, lack of a clear definition for the extra-thoracic features has been a criticism of the criteria [28]. In the study by Oldham et al., the absence of air-trapping or mosaic attenuation on HRCT and FEV1/FVC $<70 \%$ of predicted values on PFT was taken as obstructive airway disease and an FVC/DLCO $>1.6$ as unexplained pulmonary vasculopathy, particularly in non-smokers $[15,29,30]$. In the present study, a PFT was available for 24 patients; reports suggested a restrictive lung disease with a mean FEV1/FVC of $105.9 \pm 11.9$ (\% of predicted values). None of the patients' PFT suggested an obstructive airway disease. Eleven patients could not perform a PFT satisfactorily, either due to poor efforts or because of severe disease. A successful PFT requires an efficient instructor and a co-operative, 
compliant and able patient to meet the acceptability and reproducibility criteria.

A significant number of patients (60\%) had PAH, compared to the studies by Avala et al. (46\%) and Ahmad et al. $(22 \%)[19,22]$. This disparity could be due to the application of a recent definition of $\mathrm{PAH}$, with a lower threshold of $20 \mathrm{mmHg}$ in our cohort, as against the previous value of 25 $\mathrm{mmHg}$ [13]. According to the old criteria, 19 patients (54\%) had PAH. Pulmonary artery enlargement on HRCT was seen in seven patients and all of them had moderate to severe PAH. Three patients with relatively mild lung involvement and UIP pattern on HRCT were observed to have PAH. The exact contribution of ILD versus autoimmune inflammation in the pathogenesis of PAH in these patients could not be ascertained. The mean FVC and DLCO in our patients was comparable to that of other studies [15, 19, 22].

A positive ANA was the most common serologic finding in the present study, followed by anti Ro52 and anti tRNA synthetase. ANA positivity and anti Ro52 were also the most common serologic features in the cohort from Greece [25]. ANA, when repeated at 1:320 dilution, also yielded positive results, with slight change in intensity and an occasional unmasking of a different pattern. The most common pattern on ANA was fine speckled followed by homogenous and cytoplasmic. In the IPAF criteria, ANA of any pattern other than the nucleolar and centromere patterns, have to be confirmed in a titer of 1:320, irrespective of their initial intensity of fluorescence. However, we observed that if ANA is strongly positive at $1: 100$ dilution $(3+$ or $4+)$, the dilution which is routinely used when screening for CTD, it is unlikely to become negative at 1:320 dilution.

Anti-Ro52 is known to be associated with SLE, Sjogren's syndrome, myositis, autoimmune liver diseases and scleroderma. There are recent reports of isolated Ro52 being associated with ILD, excluding patients with scleroderma, and it is now being projected as a sensitive marker for ILD in patients with undifferentiated CTD like manifestations [31, 32]. It is postulated that anti-Ro52 targets many transcription factors related to the IL-17- Th-23 pathway, thus causing tissue specific inflammation and injury [33]. The present study affirms the association of anti-Ro52 with ILD in patients without any defined CTD. Ten patients in our cohort with anti-Ro52 tended to have severe lung disease, with a shorter duration of illness and displayed RP more frequently. AntiRo52 in other cohorts was seen to be associated with sicca symptoms, with few patients developing Sjogren's syndrome on follow-up [23, 25].

The demographic, clinical, serological, and morphological characteristics of IPAF patients included in the present study are compared with other recently published studies in Table 5.

Anti-tRNA synthetase antibodies are characteristically found in antisynthetase syndrome (ASS). The inclusion of such specific antibodies into the IPAF criteria has received criticism as an early ASS may be misdiagnosed as ILD only, by an unsuspecting clinician. Yamakawa et al. studied 88 patients with antisynthetase antibodies. Of these, 45 fulfilled the criteria for IPAF alone as given by ATS/ERS, while the remainder fulfilled the criteria for ASS with myositis [34]. Of the 35 patients in the current study, 8 patients (23\%) had anti-tRNA synthetase antibodies, without satisfying the criteria for ASS. NSIP was the most common radiologic pattern seen in these patients with a variable degree of severity, similar to other published studies. Anti-Jo1 was the most common antisynthetase antibody and cytoplasmic pattern was detected on ANA in two of the eight patients. ILD associated with antisynthetase antibodies is known to have good response to immunosuppression [34-36]. In the present study, patients with antisynthetase antibody had a stable outcome till the end of follow-up, with no deaths reported.

RF positivity, (more than twice the ULN) reported as one of the common serologic findings in several IPAF and autoimmune-ILD cohorts, was reported in only one patient [16, $18,19,37]$. Anti-CCP antibody is considered $98 \%$ specific for RA [38, 39]. Fischer et al. studied patients with lung disease and anti-CCP positivity, without inflammatory arthritis. Of the 74 subjects included, $14 \%$ had isolated ILD and 26\% had mixed airway lung disease. Most patients had UIP pattern of involvement and 3 of the 33 patients with high-titer anti-CCP developed articular manifestations suggestive of RA, after a median follow-up of 449 days [40]. In patients who never develop RA during follow-up, whether anti-CCP positivity presents as a forme fruste of RA, manifesting as predominant lung disease or occurs as a non-specific or false positive finding, remains to be established. In the study by Ito et al., 13 patients were anti-CCP positive, most of them had NSIP pattern of involvement, with a 5-year survival of $68.5 \%$ [27]. Anti-CCP antibodies were present in five patients $(14.3 \%)$ in the current study, none of whom had inflammatory arthritis and these patients had a predominantly NSIP pattern of involvement with severe lung disease and PAH on echocardiography.

Four patients in the present study had antibodies against Scl-70, none of whom had skin thickening, while 2 of them had RP. All of them had a moderate degree of lung involvement. Anti-Scl70 is a scleroderma-associated autoantibody, known to be associated with lung disease [30]. Recently, it has been observed that IPAF patients with myositis or the scleroderma spectrum of antibodies, have both improved survival and a better response to immunosuppression [41].

Anti-SRP, an antibody first isolated in 1986 in a patient of polymyositis, has been closely related to necrotizing and treatment-refractory myositis with cardiac involvement. Few cases report association with ILD as well [42]. Although not mentioned in the 2015 IPAF criteria, we detected three patients with anti-SRP. One of them had a photosensitive 
Table 5 Comparison of clinical and autoimmune characteristics among various reported IPAF cohorts

\begin{tabular}{|c|c|c|c|c|}
\hline Author, year of study $(N)$ & Demographics $^{\mathrm{a}}$ & Autoimmune features (\%) & Serologic features (\%) & Morphologic features (\%) \\
\hline Oldham et al. 2016 (144) & $\begin{array}{l}63.2 \pm 11 \\
52 \% \text { females }\end{array}$ & $\begin{array}{l}\text { RP (27.8), IA (17.4), mechanic } \\
\text { hands (10.4) }\end{array}$ & $\begin{array}{l}\text { ANA (77.6), anti-Ro (16.6), } \\
\text { RF (13) }\end{array}$ & $\begin{array}{l}\text { UIP ( } 54.6 \text { on HRCT } \& 73.5 \text { by } \\
\text { SLB), NSIP ( } 31.9 \text { on HRCT } \\
\text { and } 22.9 \text { by SLB) }\end{array}$ \\
\hline Ahmad et al. 2017 (57) & $\begin{array}{l}64.4 \pm 14 \\
50.9 \% \text { males }\end{array}$ & RP (74.1), IA (48) & $\begin{array}{l}\text { ANA (82.4), anti-tRNA syn- } \\
\text { thetase (17), anti Ro (9.4) }\end{array}$ & $\begin{array}{l}\text { NSIP ( } 42.1 \text { on HRCT \& } 8.8 \text { by } \\
\text { SLB), NSIP with OP overlap } \\
(15.8 \text { on HRCT) }\end{array}$ \\
\hline Chartrand et al. 2016 (56) & $\begin{array}{l}54.6 \pm 10.3 \\
71.4 \% \text { females }\end{array}$ & $\begin{array}{l}\text { RP (39), Mechanic hands } \\
\quad(28.6)\end{array}$ & $\begin{array}{l}\text { ANA (48), anti-Ro (42.9), anti- } \\
\text { tRNA synthetase (35.7) }\end{array}$ & $\begin{array}{l}\text { NSIP }(57.1 \text { on HRCT and } 23 \\
\text { by SLB) }\end{array}$ \\
\hline Lim et al. 2019 (54) & $\begin{array}{l}67.9 \pm 10.5 \\
64 \% \text { females }\end{array}$ & $\begin{array}{l}\text { IA ( } 76.5) \\
\text { RP and unexplained digital } \\
\text { edema (17.6) }\end{array}$ & ANA (63.3), RF (28.6) & $\operatorname{NSIP}(87.2)$ \\
\hline Karampeli et al. 2020 (39) & $\begin{array}{l}63.2 \pm 11 \\
69.2 \% \text { females }\end{array}$ & IA (82), rash (54), RP (25.6) & ANA (59), anti-Ro (21) & $\begin{array}{l}\text { NSIP (61.5), UIP (18) and OP } \\
(5.1)\end{array}$ \\
\hline Ito et al. 2017 (68) & $\begin{array}{l}68 \\
57.8 \% \text { females }\end{array}$ & - & SSc specific antibodies (36.7) & NSIP (64), UIP (1) and OP (20) \\
\hline Avala et al. 2020 (30) & $\begin{array}{l}52.5 \pm 14.5 \\
86.6 \% \text { females }\end{array}$ & $\begin{array}{l}\text { IA (66.7), RP and digital ulcers } \\
\text { (16.6) }\end{array}$ & ANA (60), RF (50) & $\begin{array}{l}\text { NSIP (66.7), OP (16.7) and UIP } \\
\quad(10)\end{array}$ \\
\hline Sebastian et al. 2020 (52) & $\begin{array}{l}68 \pm 14 \\
65.5 \% \text { females }\end{array}$ & IA & ANA (72.3) & $\begin{array}{l}\text { UIP (44.2), NSIP (32.7) and OP } \\
\text { (15.4) }\end{array}$ \\
\hline Present study (35) & $\begin{array}{l}50.6 \pm 13 \\
83 \% \text { females }\end{array}$ & IA (31), RP (31) & $\begin{array}{l}\text { ANA (97.4) } \\
\text { Anti Ro 52(28.5) } \\
\text { Anti t RNA (23) }\end{array}$ & NSIP (77), UIP (20) and LIP (3) \\
\hline
\end{tabular}

$N$ sample size, $I A$ inflammatory arthritis, $R P$ Raynaud's phenomenon, $A N A$ antinuclear antibody, $R F$ rheumatoid factor, $N S I P$ non-specific interstitial pneumonia, UIP usual interstitial pneumonia, $O P$ organizing pneumonia, LIP lymphocytic interstitial pneumonia., $H R C T$ high-resolution computerized tomography, SLB surgical lung biopsy

${ }^{\mathrm{a}}$ Includes age in years, mean $\pm \mathrm{SD}$ and predominant gender affected $(\%)$

skin rash and other had raised muscle enzymes without muscle weakness. Two patients had mild ILD, and one had severe ILD who ultimately succumbed to his illness.

Anti-Mi-2b is a myositis-specific antibody found in around $10 \%$ patients of dermatomyositis (DM), associated with various skin manifestations of DM. Current literature indicates that chances of a clinically significant ILD or cancer is low in DM patients with anti-Mi-2 antibodies [43, 44]. The ATS/ERS consensus statement on IPAF does not mention anti-Mi-2 antibody as one of the serologic features, but anti-Mi2b antibodies were isolated in four patients in the present study. All four patients were also positive for anti-tRNA synthetase antibodies. Whether this is a co-incidental finding or has clinical significance with respect to its association with ILD, can only be answered by multicentric prospective studies.

There is no definite consensus or guidelines available to treat IPAF patients. The treatment strategies include immunosuppression and antifibrotics, which is an extension of the modalities used in SSc-ILD and IPF. In the retrospective study by Ito et al., corticosteroids and other immunosuppressive agents like azathioprine, cyclophosphamide and calcineurin inhibitors were the most commonly used form of treatment (48\%), while only $1 \%$ received antifibrotics alone; another $2 \%$ patients received a combination of immunosuppression and antifibrotics [27]. In our study, steroids were used in all patients and the most commonly used initial dose was $0.5 \mathrm{mg}$ per kg body weight. Secondary immunosuppression in the form cyclophosphamide and mycophenolate mofetil were commonly used, while azathioprine was used in some patients with mild disease. Cyclophosphamide was used in the dosage of $500-750 \mathrm{mg} / \mathrm{m}^{2}$ body surface area monthly pulses; mycophenolate mofetil was used in the dose of $2-3 \mathrm{~g} /$ day or as tolerated and azathioprine in the dose of $2 \mathrm{mg} / \mathrm{kg}$ body weight.

Prognosis of IPAF patients or their evolution into a definite CTD is not clearly established. Oldham et al. reported a marginally better survival of IPAF patients compared to patients of IPF, and UIP pattern on HRCT was associated with a higher mortality [15]. In the study by Ito et al., $12 \%$ of patients of IPAF progressed to a definite CTD over a follow-up of 4.5 years, and deaths occurred in $27.8 \%$ patients, which was significantly higher than that of IPF patients but lower than CTD-ILD [27]. Similarly, in the retrospective study done by Chartrand et al., the survival of IPAF patients was inferior to that of other CTD-ILD [16]. Our study was not primarily designed to look at the survival rate of IPAF patients or compare them with CTD-ILD and IPF. However, in the duration of the study, four patients expired due to disease progression and/or superadded infection (one due 
to COVID-19) whereas seven patients had progression of symptoms despite treatment. None of the patients in our study evolved into a definite CTD during the follow-up period.

Our study has some limitations. The sample size was relatively small. The havoc created by the COVID-19 pandemic was and continues to be a huge impediment to patient recruitment. The present study design did not include a longer and planned follow-up of patients. Finally, the study was not designed for a simultaneous comparison with CTDILD and IPF patients, which would have been beneficial in providing a more comprehensive analysis of IPAF patients with regards to the more established entities-CTD-ILD and IPF- and possible diagnostic and therapeutic strategies.

\section{Conclusion}

In our study cohort, IPAF, as defined by the ATS/ERS 2015 consensus committee, predominantly affected females in the age group of 50 years and above. NSIP pattern on HRCT was the most common radiologic pattern, whereas inflammatory arthritis and RP were the major autoimmune features. ANA positivity, followed by anti-Ro52 antibody, were the most common serological features observed. Patients with RP had predominant UIP pattern on HRCT thorax, whereas patients with inflammatory arthritis seemed to have stable disease and good response to immunosuppression. Clinical or serological features, not currently specified in the IPAF definition, but suggesting an autoimmune etiology exist and their significance needs to be evaluated in further multi-centric longitudinal studies.

Supplementary Information The online version contains supplementary material available at https://doi.org/10.1007/s00296-021-04883-7.

\begin{abstract}
Author contributions The conception and design of the study, AW, VA, RG and DPM. Acquisition of data-KH, PB and MM. Analysis and interpretation of data- KH, RRS, NM and AW. Drafting the articleKH. Revising it critically for important intellectual content-RRS, NM, AW, MM, PB, RG, DPM, VA, AW. Final approval of the version to be submitted-KH, RRS, NM, AW, MM, PB, RG, DPM, VA, AW. Agreement to be accountable for all aspects of the work in ensuring that questions related to the accuracy or integrity of any part of the work are appropriately investigated and resolved-KH, RRS, NM, AW, MM, PB, RG, DPM, VA, AW.
\end{abstract}

Funding Intramural research grant was received to conduct this study—document number 467/R.cell-19 dated: 24/06/2019).

Data availability The authors confirm that the data supporting the findings of this study are available within the article [and/or] its supplementary materials. Further clarifications as required will be available from the corresponding author, $\mathrm{AW}$, upon reasonable request.

\section{Declarations}

Ethics approval The study was approved from the institutional ethics committee of King George's Medical University, Lucknow, India (Ethics committee approval number 377/Ethics/19.Date of approval: 02-04-19).

Consent to participate All study participants signed informed consent before enrolment.

Consent for publication All authors consent to publication and grant the Publisher exclusive license of the full copyright.

\section{References}

1. American Thoracic Society/European Respiratory Society (2002) International multidisciplinary consensus classification of the idiopathic interstitial pneumonias: this joint statement of the American Thoracic Society (ATS), and the European Respiratory Society (ERS) was adopted by the ATS Board of Directors, June 2001 and by The ERS Executive Committee, June 2001. Am J Respir Crit Care Med 165:277-304

2. Haslam PL, Thompson B, Mohammed I, Townsend PJ, Hodson ME, Holborow EJ et al (1979) Circulating immune complexes in patients with cryptogenic fibrosing alveolitis. Clin Exp Immunol 37:381-390

3. Haslam P, Turner-Warwick M, Lukoszek A (1975) Antinuclear antibody and lymphocyte responses to nuclear antigens in patients with lung disease. Clin Exp Immunol 20:379-395

4. Vij R, Noth I, Strek ME (2011) Autoimmune-featured interstitial lung disease. Chest 140:1292-1299

5. Fischer A, West SG, Swigris JJ, Brown KK, du Bois RM (2010) Connective tissue disease-associated interstitial lung disease. Chest 138:251-256

6. Corte TJ, Copley SJ, Desai SR, Zappala CJ, Hansell DM, Nicholson AG et al (2012) Significance of connective tissue disease features in idiopathic interstitial pneumonia. Eur Respir J 39:661-668

7. Fischer A, Antoniou KM, Brown KK, Cadranel J, Corte TJ, du Bois RM et al (2015) An official European Respiratory Society/ American Thoracic Society research statement: interstitial pneumonia with autoimmune features. Eur Respir J 46:976-987

8. Aletaha D, Neogi T, Silman AJ, Funovits J, Felson DT, Bingham CO et al (2010) 2010 Rheumatoid arthritis classification criteria: an American college of rheumatology/European league against rheumatism collaborative initiative. Arthritis Rheum 62:2569-2581

9. Wollheim FA (2005) Classification of systemic sclerosis. Visions and reality Rheumatol Oxf Engl 44:1212-1216

10. Aringer M, Costenbader K, Daikh D, Brinks R, Mosca M, Ramsey-Goldman R et al (2019) 2019 European league against rheumatism/American college of rheumatology classification criteria for systemic lupus erythematosus. Arthritis Rheumatol 71:1400-1412

11. Shiboski CH, Shiboski SC, Seror R, Criswell LA, Labetoulle M, Lietman TM et al (2017) 2016 ACR-EULAR classification criteria for primary Sjögren's syndrome: a consensus and data-driven methodology involving three international patient cohorts. Arthritis Rheumatol Hoboken NJ 69:35-45

12. Bottai M, Tjärnlund A, Santoni G, Werth VP, Pilkington C, de Visser M et al (2017) EULAR/ACR classification criteria for adult and juvenile idiopathic inflammatory myopathies their 
major subgroups: a methodology report. RMD Open. https:// doi.org/10.1136/rmdopen-2017-000507

13. Simonneau G, Montani D, Celermajer DS, Denton CP, Gatzoulis MA, Krowka M et al (2019) Haemodynamic definitions and updated clinical classification of pulmonary hypertension. Eur Respir 53:1801913. https://doi.org/10.1183/13993003. 01913-2018

14. Levy PT, Patel MD, Groh G, Choudhry S, Murphy J, Holland MR et al (2016) Pulmonary artery acceleration time provides a reliable estimate of invasive pulmonary hemodynamics in children. J Am Soc Echocardiogr 29:1056-1065

15. Oldham JM, Adegunsoye A, Valenzi E, Lee C, Witt L, Chen L et al (2016) Characterisation of patients with interstitial pneumonia with autoimmune features. Eur Respir J 47:1767-1775

16. Chartrand S, Swigris JJ, Stanchev L, Lee JS, Brown KK, Fischer A (2016) Clinical features and natural history of interstitial pneumonia with autoimmune features: a single center experience. Respir Med 119:150-154

17. Sharma R, Talwar D, Tuteja M (2016) Clinico-radiological and autoimmune profile correlation in patients with autoimmune featured interstitial lung disease (AIF-ILD): an observational study in Indian scenario. Chest 149:A456

18. Lim JU, Gil BM, Kang HS, Oh J, Kim YH, Kwon SS (2019) Interstitial pneumonia with autoimmune features show better survival and less exacerbations compared to idiopathic pulmonary fibrosis. BMC Pulm Med 19:120. https://doi.org/10.1186/ s12890-019-0868-9

19. Avala RC, Narahari NK, Kapoor A, Kakarla B, Varma R, Gongati PK (2020) Interstitial pneumonia with autoimmune features-an observational study in a tertiary care institute from south India. Indian J Respir Care 9:209-215

20. Kinder BW, Collard HR, Koth L, Daikh DI, Wolters PJ, Elicker B et al (2007) Idiopathic nonspecific interstitial pneumonia: lung manifestation of undifferentiated connective tissue disease? Am J Respir Crit Care Med 176:691-697

21. Carvajalino S, Reigada C, Johnson MJ, Dzingina M, Bajwah S (2018) Symptom prevalence of patients with fibrotic interstitial lung disease: a systematic literature review. BMC Pulm Med [Internet]. https://www.ncbi.nlm.nih.gov/pmc/articles/PMC59 64639. Accessed 12 Dec 2020

22. Ahmad K, Barba T, Gamondes D, Ginoux M, Khouatra C, Spagnolo P et al (2017) Interstitial pneumonia with autoimmune features: clinical, radiologic, and histological characteristics and outcome in a series of 57 patients. Respir Med 123:56-62

23. Sebastiani M, Cassone G, De Pasquale L, Cerri S, Della Casa G, Vacchi $C$ et al (2020) Interstitial pneumonia with autoimmune features: a single center prospective follow-up study. Autoimmun Rev. https://doi.org/10.1016/j.autrev.2019.102451

24. Kim EJ, Collard HR, King TE (2009) Rheumatoid arthritisassociated interstitial lung disease. Chest 136:1397-1405

25. Karampeli M, Thomas K, Flouda S, Chavatza A, Nikolopoulos D, Pieta A et al (2020) Interstitial pneumonia with autoimmune features (IPAF): a single-centre, prospective study. Mediterr J Rheumatol 31:330-336

26. Fernandes L, Nasser M, Ahmad K, Cottin V (2019) Interstitial pneumonia with autoimmune features (IPAF). Front Med 6:209. https://doi.org/10.3389/fmed.2019.00209

27. Ito $Y$, Arita M, Kumagai S, Takei R, Noyama M, Tokioka F et al (2017) Serological and morphological prognostic factors in patients with interstitial pneumonia with autoimmune features. BMC Pulm Med 17:111. https://doi.org/10.1186/ s12890-017-0453-Z

28. Oldham JM, Danoff SK (2019) COUNTERPOINT: does interstitial pneumonia with autoimmune features represent a distinct class of patients with idiopathic interstitial pneumonia? No. Chest 155:260-263
29. Steen VD, Lucas M, Fertig N, Medsger TA (2007) Pulmonary arterial hypertension and severe pulmonary fibrosis in systemic sclerosis patients with a nucleolar antibody. J Rheumatol 34:2230-2235

30. Solomon JJ, Olson AL, Fischer A, Bull T, Brown KK, Raghu G (2013) Scleroderma lung disease. Eur Respir Rev 22:6-19

31. Ferreira JP, Almeida I, Marinho A, Cerveira C, Vasconcelos C (2012) Anti-Ro52 antibodies and interstitial lung disease in connective tissue diseases excluding scleroderma. ISRN Rheumatol. https://doi.org/10.5402/2012/415272

32. Singh Y, Patil P, Longhurst H, Clarke G, Dasgupta B (2013) Isolated Anti-Ro52 Antibody - Significance and Clinical Association. ACR Meeting Abstracts. A2037. https://acrabstracts. org/abstract/isolated-anti-ro52-antibody-significance-and-clini cal-association. Accessed 6 Dec 2020.

33. Espinosa A, Dardalhon V, Brauner S, Ambrosi A, Higgs R, Quintana FJ et al (2009) Loss of the lupus autoantigen Ro52/ Trim21 induces tissue inflammation and systemic autoimmunity by dysregulating the IL-23-Th17 pathway. J Exp Med 206:1661-1671

34. Yamakawa H, Hagiwara E, Kitamura H, Iwasawa T, Otoshi R, Aiko $\mathrm{N}$ et al (2018) Predictive factors for the long-term deterioration of pulmonary function in interstitial lung disease associated with anti-aminoacyl-tRNA synthetase antibodies. Respiration 96:210-221

35. Tzilas V, Tzouvelekis A, Bouros E, Bouros D (2018) Prognosis of interstitial lung disease associated with anti-aminoacyltRNA synthetase antibodies: look in the middle. Respiration 96:207-209

36. Friedman AW, Targoff IN, Arnett FC (1996) Interstitial lung disease with autoantibodies against aminoacyl-tRNA synthetases in the absence of clinically apparent myositis. Semin Arthritis Rheum 26:459-467

37. Ferri C, Manfredi A, Sebastiani M, Colaci M, Giuggioli D, Vacchi $C$ et al (2016) Interstitial pneumonia with autoimmune features and undifferentiated connective tissue disease. Autoimmun Rev 15:61-70

38. Avouac J, Gossec L, Dougados M (2006) Diagnostic and predictive value of anti-cyclic citrullinated protein antibodies in rheumatoid arthritis: a systematic literature review. Ann Rheum Dis 65:845-851

39. Majka DS, Deane KD, Parrish LA, Lazar AA, Baron AE, Walker CW et al (2008) The duration of pre-clinical rheumatoid arthritis-related autoantibody positivity increases in subjects with older age at time of disease diagnosis. Ann Rheum Dis 67:801-807

40. Fischer A, Solomon JJ, du Bois RM, Deane KD, Olson AL, Fernandez-Perez ER et al (2012) Lung disease with anti-CCP antibodies but not rheumatoid arthritis or connective tissue disease. Respir Med 106:1040-1047

41. Wilfong EM, Schroeder G, Barnado A, Annapureddy N, Crofford LJ, Dudenhofe RB (2020) Myositis and systemic sclerosis spectrum IPAF patients are more likely to respond favorably to immunosuppression. C22 ILD THERAPY III/RAPiD: rapid abstract poster discussion session. Am J Respir Crit Care Med 201:A4561. https://www.atsjournals.org/doi/pdf/https://doi. org/10.1164/ajrccmconference.2020.201.1_MeetingAbstracts. A4561. Accessed 12 Dec 2020

42. Kao AH, Lacomis D, Lucas M, Fertig N, Oddis CV (2004) Antisignal recognition particle autoantibody in patients with and patients without idiopathic inflammatory myopathy. Arthritis Rheum 50:209-215

43. Satoh M, Tanaka S, Ceribelli A, Calise SJ, Chan EKL (2017) A comprehensive overview on myositis-specific antibodies: new and old biomarkers in idiopathic inflammatory myopathy. Clin Rev Allergy Immunol 52:1-19 
44. Targoff IN, Reichlin M (1985) The association between Mi-2 antibodies and dermatomyositis. Arthritis Rheum 28:796-803
Publisher's Note Springer Nature remains neutral with regard to jurisdictional claims in published maps and institutional affiliations. 